Under consideration for IMA Journal of Applied Mathematics

\title{
Deformation of an elastic cell in a uniform stream and in a circulatory flow
}

\author{
M. G. BLYTH AND E. I. PĂRĂU \\ School of Mathematics, University of East Anglia, \\ Norwich Research Park, Norwich, NR4 7TJ, UK.
}

[Received on 18 February 2005]

\begin{abstract}
The deformation of a circular, inextensible elastic cell is examined when the cell is placed into two different background potential flows: a uniform stream and a circulatory flow induced by a point vortex located inside the cell. In a circulatory flow a cell may deform into a mode $m$ shape with $m$-fold rotational symmetry. In a uniform stream, shapes with 2 -fold rotational symmetry tend to be selected. In a weak stream a cell deforms linearly into an ellipse with either its major or its minor axis aligned with the oncoming flow. This marks an interesting difference with a bubble with constant surface tension in a uniform stream, which can only deform into a mode 2 shape with its major axis perpendicular to the stream (Vanden-Broeck \& Keller 1980b). In general, as the strength of the uniform stream is increased from zero, solutions emerge continuously from the cell configurations in quiescent fluid found by Flaherty et al. (1972). A richly-populated solution space is described with multiple solution branches which either terminate when a cell reaches a state with a point of self-contact or loop round to continuously connect two different possible cell states under identical conditions in the absence of flow.
\end{abstract}

Keywords: Inviscid flow, fluid-structure interaction, elastic cell

\section{Introduction}

Many applications in engineering and the physical and biological sciences involve the interaction between a moving fluid and a solid object. Of particular interest is the case when a free boundary is present, for example when an elastic solid deforms in response to local fluid forces (see, for example, Korobkin et al. 2011). Examples include flapping flags (e.g. Michelin \& Llewellyn-Smith 2010), the swimming of microorganisms (e.g. Lauga \& Powers 2009), and the transport of liquified natural gas (e.g. Ten et al. 2011). Knowledge of how an elastic boundary deforms in the presence of a class of simple fluid flows is of fundamental importance. For example, recent work by Alben (2011) examines the interaction between a point vortex and a flexible wall. In the present paper we study the equilibrium states which may be achieved when an elastic cell is placed into two prototype fluid motions, a uniform stream and a swirling flow.

In a seminal paper, Flaherty et al. (1972) calculated the buckled states of a thin-walled elastic cell subjected to a constant transmural pressure, that is a constant net pressure between the exterior of the cell and the interior. They showed that when the transmural pressure exceeds a sequence of threshold values, the cell may buckle into a configuration with two-fold, three-fold, etc., rotational symmetry. As the transmural pressure is increased, cells eventually reach a state with a point of self-contact, and further increasing the transmural pressure produces cells whose profiles self-intersect. Flaherty et al. (1972) bypassed this difficulty, and widened the space of physically admissible solutions, by allowing the contact point to extend into a line of self-contact which can be described using a similarity solution structure.

When an elastic cell is placed into a moving fluid, the local transmural pressure is not constant but 
varies around the cell boundary. In this case, the problem of predicting the cell shape is inherently coupled to the exterior flow problem through the dynamic balance of fluid and elastic stresses at the cell boundary. In the present work, we assume that the exterior fluid motion is inviscid and irrotational and that the pressure inside the cell is constant. Our goal is to predict the possible equilibrium cell shapes. Two types of exterior flow field are examined: first a uniform stream and second the circulatory flow induced by a point vortex placed inside the cell. In each case the problem is solved by seeking an analytic description for the complex velocity potential in the form of a power series in an appropriate conformally-mapped domain. The coefficients of the series are determined numerically by enforcing the dynamic stress condition on the cell boundary via a collocation method. Our work complements studies on the similar problem involving a bubble with surface tension conducted by Vanden-Broeck \& Keller (1980b) for a bubble placed in a uniform stream, and by Crowdy (1999a) and Wegmann \& Crowdy (2000) for a bubble placed into a flow with circulation. Other related work includes studies of inviscid waves on a fluid annulus by Crowdy (1999b), Crowdy (2001) and Blyth \& Vanden-Broeck (2005).

In the next section we formulate the general framework for studying the deformation of the cell in either of the two mentioned flow-fields. Section 3 covers the case of a uniform flow field, and section 4 addresses the circulatory flow problem.

\section{General formulation}

We consider the deformation of a closed, two-dimensional elastic cell placed into an inviscid, irrotational flow. Two exterior flow-fields are considered, namely a uniform stream and a circulatory flow induced by a point vortex located inside the cell. The two different flow configurations are illustrated in Figures 1 and 8 respectively. In both cases, the fluid occupies the infinite region exterior to the cell and the interior region is assumed to be held at a constant pressure $p_{a}$. The cell boundary is modelled as a thin elastic shell.

Ignoring inertia, a balance of forces and moments in the elastic cell boundary requires (e.g. Flaherty et al. 1972)

$$
\gamma^{\prime}+\kappa q=0, \quad q^{\prime}-\kappa \gamma=p_{a}-p, \quad q=m^{\prime},
$$

where $\gamma(s)$ and $q(s)$ are the tangential and shear tensions respectively with units of force per unit length, $m(s)$ is the bending moment, $p$ is the pressure in the liquid at the cell boundary, and $s$ denotes arc distance along the cell boundary measured positively in the counterclockwise direction. The primes in (2.1) denote differentiation with respect to $s$. The curvature $\kappa=-\theta^{\prime}(s)$, where $\theta$ is the angle made between the tangent to the boundary and the horizontal. For the undeformed circular cell of radius $a$ the curvature is $\kappa=-1 / a$. For the bending moment, we adopt the simple linear relationship

$$
m=E_{B}\left(\kappa-\kappa_{R}\right),
$$

where $E_{B}$ is a bending modulus and $\kappa_{R}$ is a reference curvature attained when the elastic cell is in its resting state; in the present work $\kappa_{R}$ will be assumed to be a constant and, therefore, to play no part in the dynamics according to (2.1). According to thin shell theory, $E_{B}=E h^{3} /\left[12\left(1-v^{2}\right)\right]$, where $E$ is the Young's modulus, $v$ is the Poisson ratio, and $h$ the shell thickness which is here assumed to be constant (e.g. Fung 1965). The relationship (2.2) has been justified for the case of an inextensible elastic shell, as assumed here, by Pozrikidis (2002). Integrating (2.1) once with respect to arc length and eliminating $\gamma$ and $q$, we obtain the dynamic relationship between the liquid pressure at the cell boundary and elastic 
stresses,

$$
p_{a}-p=E_{B}\left(\kappa^{\prime \prime}+\frac{1}{2} \kappa^{3}-\sigma \kappa / L^{2}\right)
$$

where $\sigma=L^{2}\left(\gamma / E_{B}+\kappa^{2} / 2\right)$ is a dimensionless constant of integration, and $L$ is a length scale to be discussed below.

To describe the flow it is convenient to introduce a complex potential $w=\phi+\mathrm{i} \psi$, where $\phi(x, y)$ is the velocity potential and $\psi(x, y)$ is the stream function. Requiring that the complex function $w(z)$ be analytic, it only remains to satisfy the relevant boundary conditions. These stipulate that the cell boundary is a streamline and that the liquid pressure at the cell boundary is in balance with the elastic forces according to (2.1). Starting from Bernoulli's equation, and making use of (2.3), we non-dimensionalise using $L$ as a reference length and $U$ as a reference speed associated with the background flow to obtain

$$
\frac{1}{2} \alpha|\mathbf{u}|^{2}-\left(\kappa^{\prime \prime}+\frac{1}{2} \kappa^{3}-\sigma \kappa\right)=B
$$

on the cell boundary, where $|\mathbf{u}|=|\nabla \phi|$ is the flow speed, $B$ is the Bernoulli constant, and

$$
\alpha=\frac{\rho L^{3} U^{2}}{E_{B}},
$$

where $\rho$ is the density of the liquid. The particular choices for $U$ and $L$ will be discussed in subsequent sections.

In the limit $\alpha \rightarrow \infty$, with $\alpha / \sigma=O(1)$, the first two terms in the bracket on the left hand side of (2.4) are effectively removed, and we recover the problem for a bubble with surface tension in an ambient flow. In this case, for the circulatory flow problem, analytical solutions have been derived by Crowdy (1999a) and Wegmann \& Crowdy (2000). An exact solution for a bubble in a uniform flow was obtained by McLeod (1955); numerical solutions were subsequently presented by Petrova (1970) and Vanden-Broeck \& Keller (1980b).

Taking the limit $\alpha \rightarrow 0$ in (2.4) effectively removes the first term leaving the classical problem of an elastic shell exposed to a constant pressure difference across its boundary (e.g. Antman 2005). This limit can be thought of as removing the background flow by setting $U$ to zero so that the pressure is uniform throughout the liquid. A simple solution is given by $\kappa=$ constant corresponding to a circular shell. In this case the constant $B$ on the right hand side of (2.4) represents the transmural pressure across the shell boundary $\Delta p$ (= exterior pressure minus interior pressure). Classical theory predicts that a circular cylindrical shell buckles into a non-circular shape with $n$-fold rotational symmetry, for integer $n$, when the transmural pressure across the shell boundary $\Delta p$ exceeds the critical value $\Delta p=n^{2}-1$ (e.g. Fung 1997, p. 217). Flaherty et al. (1972) studied post-buckling solutions valid when $\Delta p>n^{2}-1$ including shapes with points of contact and segments of contact.

\section{An elastic cell in a uniform stream}

First we examine the deformation experienced by a cell placed in a uniform stream of strength $U_{\infty}$, as is illustrated in Figure 1 $(a)$. In our calculations we assume that the cells have both a horizontal and a vertical line of symmetry, as in the sketch in Figure 1 $(a)$. To calculate the shape of the cell this we make use of the conformal mapping method of Ozugurlu \& Vanden-Broeck (2000), the particular details of which are clearly described in that paper and so we will provide only the essential details needed for the 
4 of 21

(a)

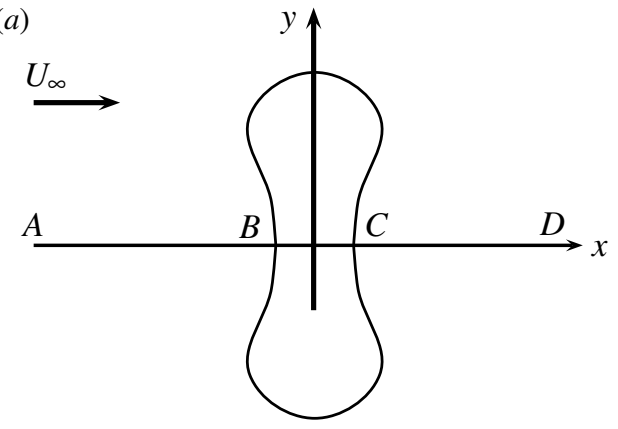

M. G. Blyth \& E. I. Părău

(b)

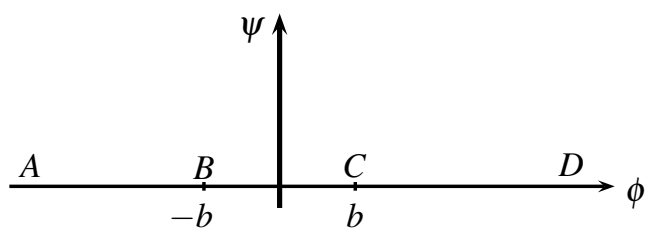

(c)

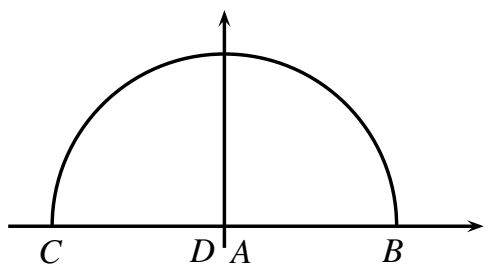

Fig. 1: (a) Sketch of uniform flow past an elastic cell in the physical $(x, y)$ plane. (b) The flow configuration in the complex potential $w=\phi+\mathrm{i} \psi$ plane. (c) The semicircular image in the $t$-plane after the conformal mapping (3.2). Points $A-D$ are mapped between the planes as shown.

present problem. The key idea is to map the flow in the upper-half complex potential $w$-plane shown in Figure 1(b) to the interior of the semicircle of unit radius in the complex $t$-plane shown in Figure 1(b). The line $\psi=0$ corresponds to a horizontal line of symmetry in the physical plane. The upper half of the elastic cell boundary occupies $-b \leqslant \phi \leqslant b$ and is mapped to the semicircular boundary in the $t$-plane.

For convenience we choose $U=U_{\infty}$ as the velocity scale and $L=b / U_{\infty}$ as the length scale. Henceforth all variables are assumed to have been made dimensionless according to these scales. The dimensionless elastic parameter (2.5) takes the form $\alpha=\rho b^{3} /\left(U_{\infty} E_{B}\right)$. Making use of the conditions at infinity, we may determine the Bernoulli constant and hence write (2.4) as

$$
\frac{1}{2} \alpha|\mathbf{u}|^{2}-\left(\kappa^{\prime \prime}+\frac{1}{2} \kappa^{3}-\sigma \kappa\right)=\frac{1}{2} \alpha+P,
$$

where $P=b^{3}\left(p_{\infty}-p_{a}\right) /\left(E_{B} U_{\infty}^{3}\right)$ and $p_{\infty}$ is the far-field pressure. On the upper part of the cell boundary, traversing from $B$ to $C$, the dimensionless velocity potential occupies the range $-1 \leqslant \phi \leqslant 1$. The conformal mapping to the $t$-plane is effected by taking

$$
-w=\frac{1+t^{2}}{2 t}
$$

Under this mapping points $A-D$ in the complex potential $w$-plane shown in Figure $1(b)$ are mapped to the $t$-plane as shown in Figure 1(c). Near to the fore and aft stagnation-points located at $B$ and $C$ in Figure 1, the complex velocity $\zeta=u-\mathrm{i} v$ has the local behaviour $\zeta \sim O\left(t^{2}-1\right)$ as $t \rightarrow \pm 1$, and for this 
reason it is convenient to seek an analytic description for the complex velocity inside the unit semicircle in the form

$$
\zeta=\left(1-t^{2}\right) \sum_{n=1}^{\infty} c_{n} t^{2 n-2},
$$

where the infinite set of coefficients $c_{n}$ is to be found. These coefficients are taken to be real in order to satisfy the kinematic condition that $v=0$ along $A B$ and $C D$ in Figure 1(a). The right hand side in (3.3) has been constructed so that $\zeta$ is analytic and that the flow is symmetric about the vertical centreline, so that on the unit semicircle

$$
\zeta\left(\mathrm{e}^{\mathrm{i} \omega}\right)=\overline{\zeta\left(\mathrm{e}^{\mathrm{i}(\pi-\omega)}\right)}
$$

for angles $0 \leqslant \omega \leqslant \pi$, where the bar indicates the complex conjugate.

In the far field the flow must match to the uniform stream, and this requires $\zeta \rightarrow 1$ as $t \rightarrow 0$, whereupon

$$
c_{1}=1
$$

A numerical method is used to satisfy the dynamic boundary condition (2.4) on the cell boundary. This is discussed in the next section.

\subsection{Numerical method}

Following Ozugurlu \& Vanden-Broeck (2000) we satisfy the dynamic boundary condition approximately using a collocation method. We discretize the semicircular boundary in the $t$-plane by writing $t=\mathrm{e}^{\mathrm{i} \omega}$ and taking $N$ equally-spaced points in $\omega$ in the range $0<\omega<\pi / 2$ excluding the end-points 0 and $\pi / 2$. Noting that

$$
x_{\phi}+\mathrm{i} y_{\phi}=\frac{1}{u-\mathrm{i} v}=\frac{u+\mathrm{i} v}{u^{2}+v^{2}},
$$

after a little algebra we may obtain the convenient expression for the curvature,

$$
\kappa=\frac{1}{\sin \omega} \frac{u v_{\omega}-v u_{\omega}}{\left(u^{2}+v^{2}\right)^{1 / 2}},
$$

where we have used the fact that $\phi=-\cos \omega$ according to the mapping (3.2). It should be noted that according to (3.7) the undeformed circular cell has negative curvature. A closed form expression for the term $\kappa^{\prime \prime}$ may be readily derived; however it is rather lengthy and it is suppressed in the interest of brevity. Fixing two out of the three parameters $\alpha, P$ and $\sigma$, we truncate the infinite series in (3.3) at the finite level $n=N$ and apply the dynamic boundary condition (2.4) at each of the $N$ collocation points to obtain $N$ nonlinear algebraic equations for the $N$ unknowns $c_{2}, \ldots, c_{N}$ and the remaining parameter. These equations are solved using Newton iteration supplied with a suitable initial condition.

Although it provides a convenient means for performing the computations, using $b / U_{\infty}$ as the reference length scale makes physical interpretation difficult. To present our results we will first rescale using the fixed cell perimeter as the reference length. To do this we first compute the dimensional perimeter of the cell $\lambda\left(b / U_{\infty}\right)$, where $\lambda$ is the dimensionless cell perimeter defined by

$$
\lambda=2 \int_{-b}^{b}\left(u^{2}+v^{2}\right)^{-1 / 2} \mathrm{~d} \phi .
$$


We then report results with reference to the new dimensionless parameters $\hat{\alpha}, \hat{P}$ and $\hat{\sigma}$, where

$$
\hat{\alpha}=\left(\frac{\lambda}{2 \pi}\right)^{3} \alpha=\frac{\rho a^{3} U_{\infty}^{2}}{E_{B}}, \quad \hat{P}=\left(\frac{\lambda}{2 \pi}\right)^{3} P=\frac{a^{3}\left(p_{\infty}-p_{a}\right)}{E_{B} U_{\infty}^{3}}, \quad \hat{\sigma}=\left(\frac{\lambda}{2 \pi}\right)^{2} \sigma .
$$

Cells are then plotted in units of $a$, the equivalent radius of a circle with the same physical perimeter as the cell, rather than units of $b / U_{\infty}$. We note that $2 \pi a=\lambda b / U_{\infty}$. A useful measure for characterising the deformed cells is the dimensionless bending energy

$$
\hat{\mathscr{E}}=\frac{1}{2 \pi} \int_{0}^{2 \pi} \hat{\kappa}^{2} \mathrm{~d} \hat{s}
$$

where $\hat{\kappa}=(\lambda / 2 \pi) \kappa$ and $\hat{s}=(2 \pi / \lambda) s$. The dimensionless bending energy $\hat{\mathscr{E}}$ has been normalised so that it is equal to unity for a circular cell. For a typical calculation, we fix $\hat{\alpha}$ and $\hat{P}$ and obtain $\hat{\sigma}$ as part of the solution.

Under the new non-dimensionalisation the dynamic boundary condition (3.1) becomes

$$
\frac{1}{2} \hat{\alpha}|\mathbf{u}|^{2}-\left(\hat{\kappa}^{\prime \prime}+\frac{1}{2} \hat{\kappa}^{3}-\hat{\sigma} \hat{\kappa}\right)=\frac{1}{2} \hat{\alpha}+\hat{P},
$$

where a prime denotes differentiation with respect to $\hat{s}$. When $\hat{\alpha}=0,(3.11)$ reduces precisely to the equation studied by Flaherty et al. (1972) for an elastic cell in a quiescent environment. Multiple solutions exist for this problem. First, a circular cell is always a solution. Bifurcations from the circular state occur at a sequence of critical pressures, $\hat{P}=n^{2}-1$, for natural number $n$, whereupon the cell can buckle into a non-circular shape with $n$-fold rotational symmetry. These buckled shapes eventually reach a pinched state in which the cell has at least one point of self-contact. Flaherty et al. (1972) computed the first few critical pressures at which pinching occurs for mode 2, 3 and 4 shapes to be

$$
\hat{P}_{c 2}=5.247, \quad \hat{P}_{c 3}=21.65, \quad \hat{P}_{c 4}=51.84 .
$$

Given the expected left-right and up/down symmetry of the cells placed into a uniform stream, we are only interested in the even mode buckled states with $n=2 k$, for natural number $k$.

\subsection{Linear theory: $\alpha \ll 1$.}

Vanden-Broeck \& Keller (1980b) conducted a linearised analysis for a bubble in a uniform stream in the limit when the bubble is only mildly deformed. To a first order approximation, they found the bubble deforms into the shape of an ellipse with its major axis oriented perpendicular to the oncoming stream. We may perform a similar analysis here for a circular cell placed into a weak uniform stream by taking $\hat{\alpha}$ to be small. The analysis is most conveniently presented using the nondimensionalisation introduced at the end of section 3.1, so that the dimensionless radius of the undeformed circular cell is equal to unity.

In the simplest case, we consider small perturbations from the circular state. To leading order approximation, the flow around the cell corresponds to that due to a uniform stream past a circular cylinder of unit radius, so that to a first approximation the slip velocity on the cell boundary is given by $\mathbf{u}=-2 \sin s \mathbf{e}_{\theta}$, where $\mathbf{e}_{\theta}$ is the polar unit vector tangent to the circle (e.g. Batchelor 1967). Retaining the leading order contribution from the exterior flow, the dynamic boundary condition (3.11) is written

$$
\hat{\kappa}^{\prime \prime}+\frac{1}{2} \hat{\kappa}^{3}-\hat{\sigma} \hat{\kappa}=\frac{1}{2} \hat{\alpha}-\hat{\alpha} \cos 2 s-\hat{P},
$$


which we recognise as a forced Duffing equation (e.g. Drazin 1992). Assuming that the cell is only slightly deformed from a circle, we expand by writing

$$
\hat{\kappa}(s)=-1+\hat{\alpha} \hat{\kappa}_{1}(s)+\cdots, \quad \hat{\sigma}=\sigma_{0}+\hat{\alpha} \sigma_{1}+\cdots .
$$

Substituting these expansions into (3.13), we obtain at leading order $\hat{P}=1 / 2-\sigma_{0}$. At $O(\hat{\alpha})$ we find that $\sigma_{1}=1 / 2$ and that $\hat{\kappa}_{1}$ satisfies the equation

$$
\hat{\kappa}_{1}^{\prime \prime}+(\hat{P}+1) \hat{\kappa}_{1}=-\cos 2 s .
$$

The homogeneous form of this equation is the same as that for small perturbations from the circular state in the absence of flow. It is known that (e.g. Fung 1997, p. 217) a circular cell buckles into a shape with $n$-fold rotational symmetry when the transmural pressure exceeds a sequence of threshold values, which in the present analysis correspond to $\hat{P}=n^{2}-1$ for natural number $n$.

The right hand side of (3.15) forces a deformation with two-fold rotational symmetry. The particular integral solution to $(3.15)$ is

$$
\hat{\kappa}_{1}=\frac{1}{(3-\hat{P})} \cos 2 s
$$

which implies that the elastic cell deforms linearly into an ellipse. Of particular interest is the fact that when $\hat{P}<3$ the ellipse has its major axis oriented at ninety degrees to the oncoming stream, as for the bubble problem studied by Vanden-Broeck \& Keller (1980); however, when $\hat{P}>3$ the ellipse has its major axis aligned with the oncoming stream, a circumstance which cannot arise for a bubble. A kind of resonance occurs at the pressure $\hat{P}=3$ where (3.16) fails and no periodic solution is possible according to the present linear theory. This issue can be resolved using a weakly nonlinear theory, as is discussed in the next section.

\subsection{Weakly nonlinear theory: $\hat{P}=3$.}

In this section we use a weakly nonlinear theory to resolve the behaviour at the resonant pressure $\hat{P}=3$. The key idea is to posit an expansion valid when $\hat{\alpha} \ll 1$ which is capable of removing the secular terms which are responsible for the failure of the linear theory. We fix $\hat{P}=3$ and expand by writing

$$
\hat{\kappa}(s)=-1+\hat{\alpha}^{1 / 3} k_{1}(s)+\hat{\alpha}^{2 / 3} k_{2}(s)+\hat{\alpha} k_{3}(s)+\cdots,
$$

and

$$
\hat{\sigma}=\sigma_{0}+\hat{\alpha}^{1 / 3} \sigma_{1}+\hat{\alpha}^{2 / 3} \sigma_{2}+\cdots .
$$

Substituting into (3.11) we derive a sequence of problems at each order. These problems are to be solved subject to the conditions appropriate for mode 2 shapes (Flaherty et el. 1972)

$$
\hat{\kappa}^{\prime}(0)=\hat{\kappa}^{\prime}(\pi / 2)=0, \quad \int_{0}^{\pi / 2} \hat{\kappa} \mathrm{d} s=-\frac{\pi}{2}
$$

also being satisfied at each order of approximation. We present only the important details of the analysis. At leading order we find that $\sigma_{0}=-5 / 2$. At $O\left(\hat{\alpha}^{1 / 3}\right)$ we obtain $k_{1}=a_{1} \cos 2 s+b_{1} \sin 2 s$ for constants $a_{1}$ and $b_{1}$. Proceeding to $O(\hat{\alpha})$ we find that $\sigma_{1}=0$ and that

$$
b_{1}\left(a_{1}^{2}+b_{1}^{2}\right)=0, \quad a_{1}\left(a_{1}^{2}+b_{1}^{2}\right)=\frac{32}{9}, \quad \sigma_{2}=\frac{3}{4}\left(a_{1}^{2}+b_{1}^{2}\right) .
$$


(a)

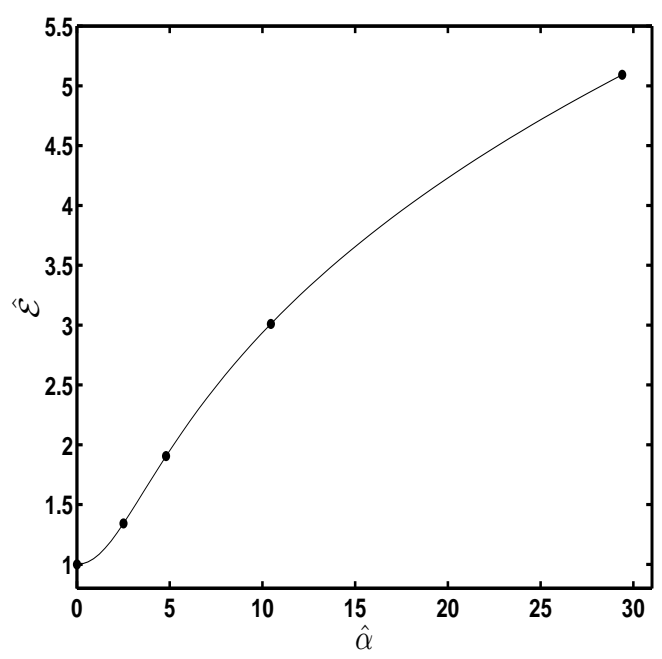

(b)

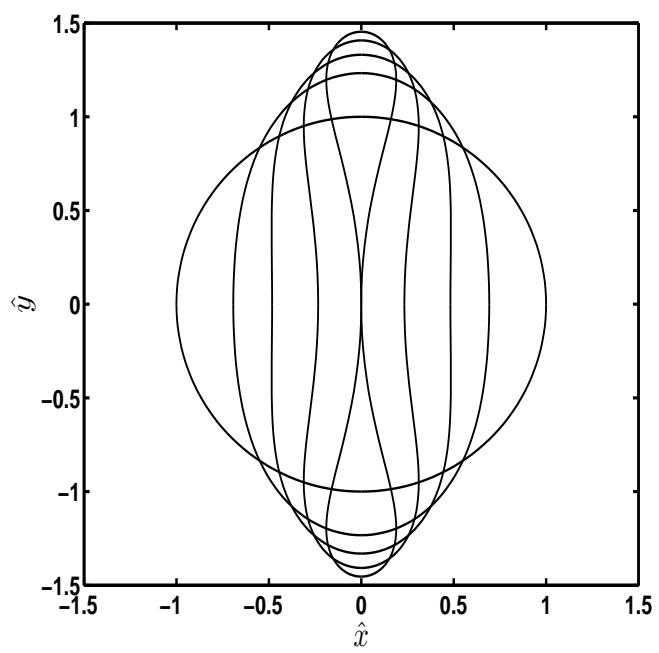

FIG. 2: (a) Solution branch showing $\hat{\mathscr{E}}$ against $\hat{\alpha}$ for $\hat{P}=0$. (b) Cell profiles along the solution branch shown in $(a)$. The cells are plotted on an $(\hat{x}, \hat{y})$ graph where $(\hat{x}, \hat{y})=(2 \pi / \lambda)(x, y)$. Each cell has the same perimeter equal to $2 \pi$.

These results follow from enforcing conditions (3.19) and from requiring that the secular terms which appear in the solution for $k_{3}(s)$ are eliminated. The solution to (3.20) is $a_{1}=(2 / 3) 12^{1 / 3}, b_{1}=0$, and $\sigma_{2}=(1 / 3) 12^{2 / 3}$. Since $a_{1}>0$ cells will deform into mode 2 shapes with their major axis perpendicular to the uniform stream. The bending energy is found to be

$$
\hat{\mathscr{E}}=1+\left(\frac{a_{1}^{2}}{2}\right) \hat{\alpha}^{2 / 3}+O(\hat{\alpha}),
$$

which implies the presence of a cusp in the $\hat{\mathscr{E}}-\hat{\alpha}$ solution curve at $\hat{P}=3$. Both of the last two observations will be corroborated by the results of full nonlinear calculations to be discussed in the next section.

\subsection{Results}

The linear theory in section 3.2 suggests that important differences will arise depending on whether we have $\hat{P}<3$ or $\hat{P}>3$. We begin by examining the former case, $\hat{P}<3$. In Figure $2(a)$ we show the solution curve in $\hat{\mathscr{E}}-\hat{\alpha}$ space for the case $\hat{P}=0$. In agreement with the linear theory, the cell starts to deform into a vertically aligned elliptical shape as $\hat{\alpha}$ is increased from zero. The branch shown in Figure 2(a) terminates at the point where the cell pinches together. Cell profiles are shown in Figure 2(b). The leftmost dot in Figure 2(a) corresponds to the circular profile in Figure 2(b). The remaining dots correspond to cell profiles in an obvious way. The calculations may be continued beyond the point of self-contact to obtain self-intersecting cell profiles. For an elastic cell in an ambient medium at constant pressure a point of contact is established when the transmural pressure between the interior and the exterior reaches a critical value, and thereafter self-intersection is found. Flaherty et al. (1972) 


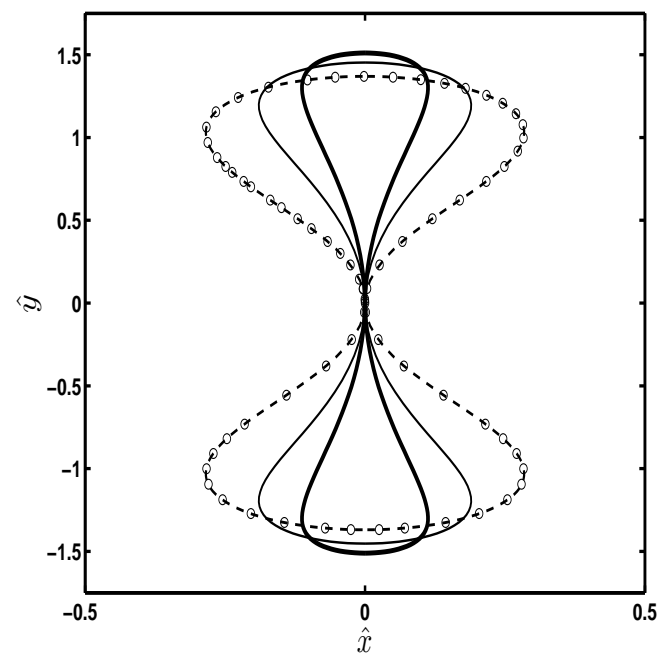

FIG. 3: Pinching cell profiles for uniform stream flow for $\left(\hat{P}, \hat{\alpha}_{c}\right)=(-200,789)$ shown with a thick solid line, $(0,29.3)$ shown with a thin solid line, and $(5.247,0)$ shown with a broken line. The circles indicate the Flaherty et al. (1972) solution for a static cell. The cells are plotted on an $(\hat{x}, \hat{y})$ graph where $(\hat{x}, \hat{y})=(2 \pi / \lambda)(x, y)$.

continued the solution space beyond the point of contact by constructing a similarity solution for which the corresponding cell profiles are made up of closed loops connected by a straight line. Working on a different problem but in a similar vein, Vanden-Broeck \& Keller (1980a) extended the solution of Crapper (1957) for capillary waves on a liquid surface beyond the point where the waves establish periodic points of self-contact enclosing trapped bubbles of air by allowing for an adaptable pressure inside the bubbles. Extending our solution space in the same spirit ought to be possible for the present problem, although we do not pursue this here.

In Figure 3 we show the limiting cell profiles with a point of self-contact for three different values of $\hat{P}$. In each case the cells were found by trial and error to identify the correct value of $\hat{\alpha}$ for selfcontact, which we call $\hat{\alpha}_{c}$. In the extreme case, $\hat{\alpha}_{c}=0$ at $\hat{P}=5.247$ which coincides precisely with the critical pressure identified by Flaherty et al. (1972) for a static cell with two-fold rotational symmetry to develop a point of self-contact. Flaherty's solution at this pressure is shown in the figure with circles. As $\hat{P}$ is reduced from this critical pressure, the point-contact cells become more slender. Indeed, as $\hat{P}$ passes through zero and then becomes large and negative, the cells become increasingly slender and eventually approach a limiting shape. The approach to a limiting cell configuration is indicated by the behaviour of the speed at the top of the cell, $q=|\mathbf{u}|^{2}$, which is shown in Figure 4(a). As the limiting shape is approached the magnitude of the elastic terms on the left hand side of the dynamic boundary condition (3.1) becomes relatively small. This suggests that the limiting profile corresponds to the pinched bubble with surface tension in a uniform stream computed by Vanden-Broeck \& Keller (1980b). Repeating their calculation for the bubble by disregarding the elastic terms in (2.4), we find that $\hat{P} / \hat{\alpha}_{c}=-0.273$ and $q=6.56$ at the top of the bubble. The latter value is shown by the dashed line in Figure $4(a)$. In Figure $4(b)$ the elastic cell profiles for large negative $\hat{P}$ clearly approach the limiting bubble shape, which is shown with circles. 
10 of 21

(a)

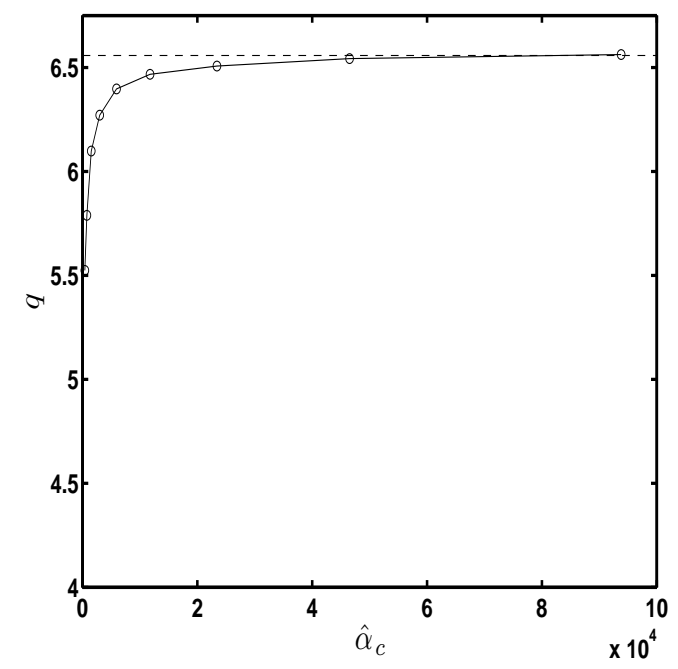

M. G. Blyth \& E. I. Părău

(b)

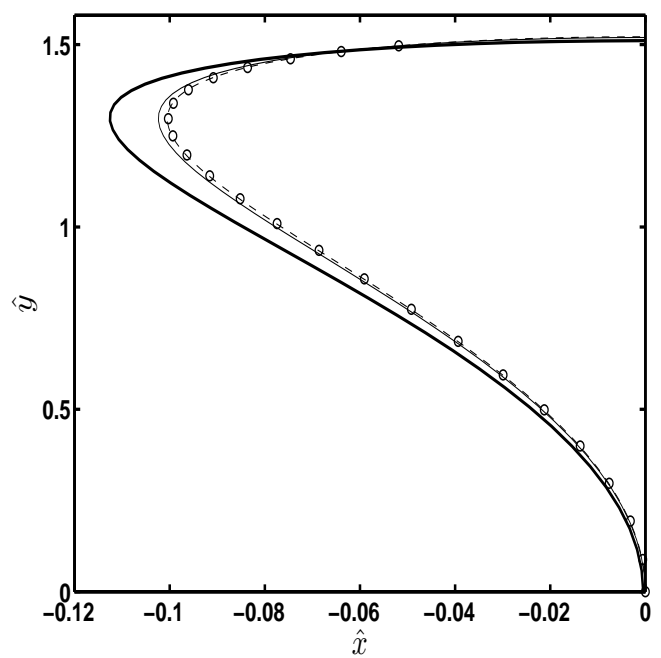

FIG. 4: (a) The speed $q=|\mathbf{u}|^{2}$ at the top of the pinched cells (as illustrated in Figure 3) versus $\hat{\alpha}_{c}$. The dashed line shows the limiting value for a pinched bubble $q=6.56$. (b) Close-up of one quarter of the pinching cell profiles for $\left(\hat{P}, \hat{\alpha}_{c}\right)=(-200,761)$ (thick solid line), $\left(-1.6 \times 10^{3}, 5.92 \times 10^{3}\right)$ (thin solid line) and $\left(-2.56 \times 10^{4}, 9.38 \times 10^{4}\right)$ (dashed line). The limiting shape corresponding to a pinched bubble is shown with circles. Note that $(\hat{x}, \hat{y})=(2 \pi / \lambda)(x, y)$.

We now turn particular attention on the case $\hat{P}>3$. As remarked above, the solution space is expected to be much richer here because of the possibility of multiple solutions at $\hat{\alpha}=0$. In Table 1 we summarise the possible cell shapes at $\hat{\alpha}=0$. Note that we are only interested in physically admissible solutions - other mathematically possible solutions exist but they correspond to cell shapes with one or more self-intersections. Since we are interested in the effect of a uniform stream on the cell shapes for $\hat{\alpha}>0$, the orientation of mode $n$ buckled states is important to within the symmetry restrictions imposed by the problem (recall that cells are required to be both up/down and left/right symmetric). With this in mind, mode 2 shapes at $\hat{\alpha}=0$ with their major axes oriented vertically and horizontally are to be treated as distinct. Similarly, there are two distinct even mode $n$ shapes that are related to each other by a rotation about their centre through an angle $\pi / n$. We emphasise that although mode- $n$ shapes are possible at $\hat{\alpha}=0$ (the Flaherty problem), when $\hat{\alpha}>0$ the mode 2 shape is forced by the background flow. However, higher order modes may emerge and possibly dominate to produce cell shapes with higher order rotational symmetry as we shall see.

\begin{tabular}{l|c|c|c|c}
$3 \leqslant \hat{P} \leqslant 5.247$ & $5.247 \leqslant \hat{P} \leqslant 15$ & $15 \leqslant \hat{P} \leqslant 35$ & $35 \leqslant \hat{P} \leqslant 51.84$ & $51.84 \leqslant \hat{P} \leqslant 63$ \\
Circle, mode 2 & Circle & Circle, mode 4 & Circle, mode 4, mode 6 & Circle, mode 6
\end{tabular}

Table 1: Possible physically admissible cell shapes at $\hat{\alpha}=0$. Note that for a mode $n$ shape. Higher mode $k$ shapes exist beyond the range shown here. 
(a)

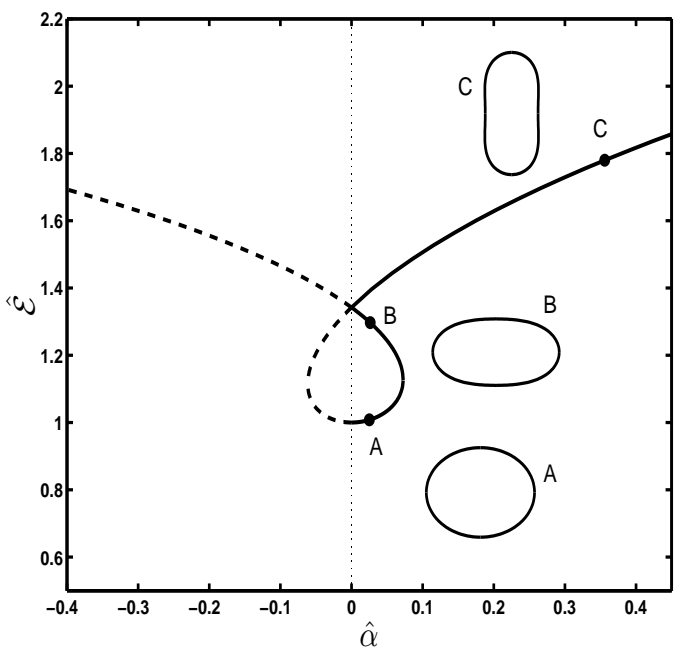

(b)

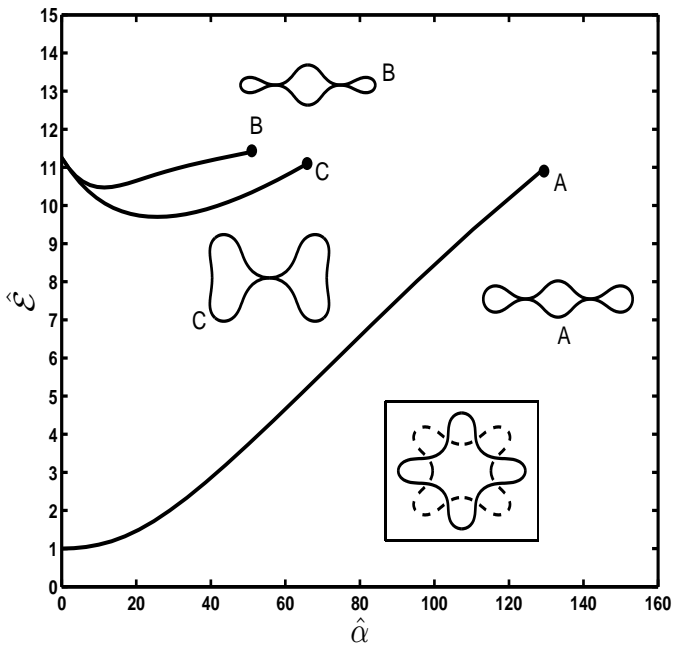

FIG. 5: (a) Solution branch for $\hat{P}=3.2$. Unphysical sections of the branch in $\hat{\alpha}<0$ are shown with a broken line. ( $b$ ) Solution branches for $\hat{P}=25$. The pinched cells corresponding to the dots at the end of each branch are also shown. The inset in $(b)$ shows the two buckled shapes at $\hat{\alpha}=0$. The solid/dashed cell in the inset deforms into cell $B / C$ as $\hat{\alpha}$ is increased.
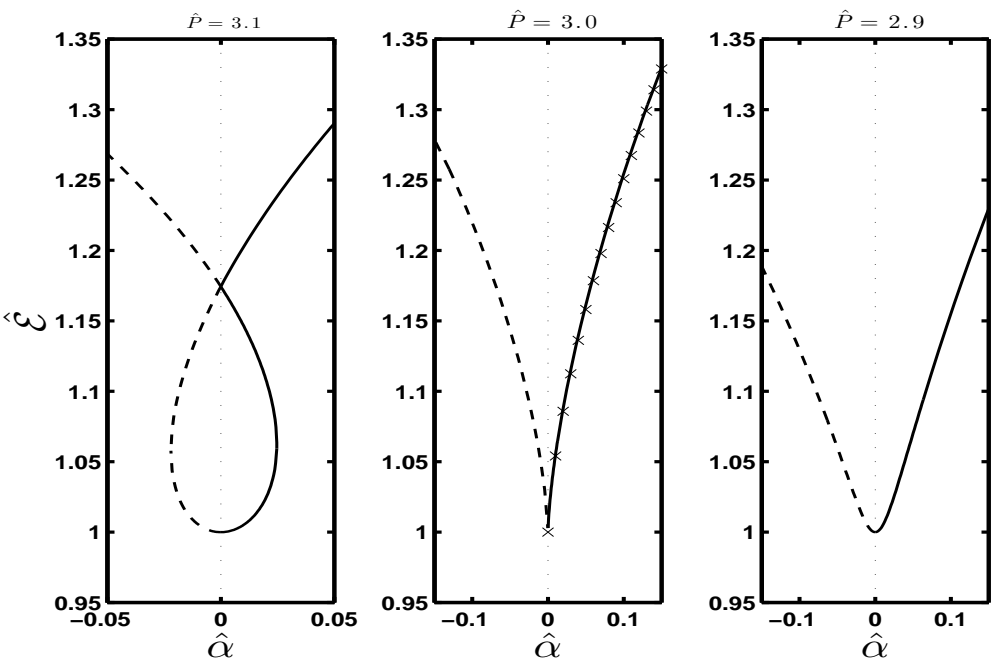

FIG. 6: The transition through a cusp at $\hat{\alpha}=0$ as the pressure parameter passes through the resonant value $\hat{P}=3$. Portions of the curves in $\hat{\alpha}<0$ corresponding to non-physical cell shapes are dashed. The crosses on the $\hat{P}=3$ curve represent the weakly nonlinear prediction $(3.21): \hat{\mathscr{E}}=1+1.16 \hat{\alpha}^{2 / 3}$. 
(a)

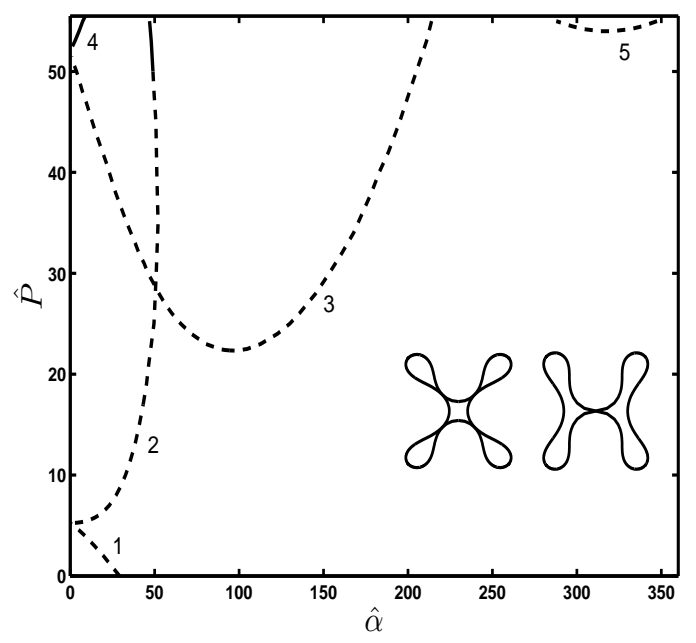

(b)

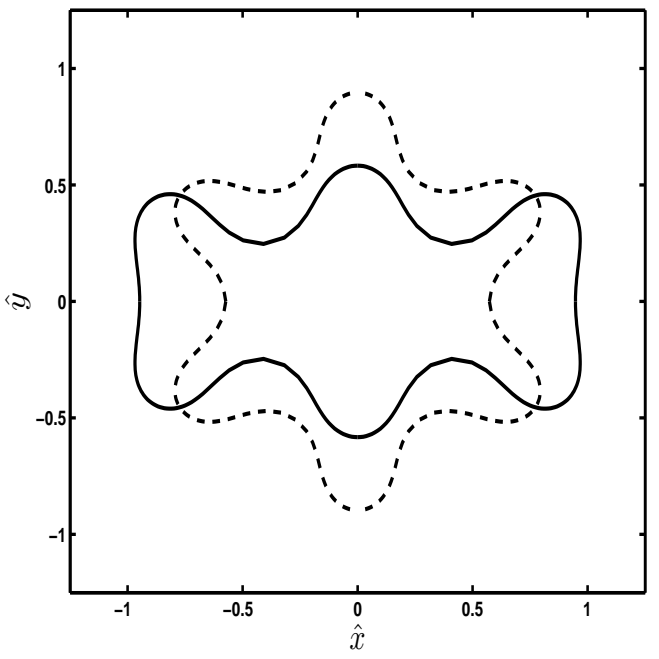

FIG. 7: (a) Solution branches corresponding to pinched cell shapes. Branches 1 and 2 emerge from $\hat{P}=5.247$ at $\hat{\alpha}=0$, and branches 2,4 emerge from $\hat{P}=51.84$ at $\hat{\alpha}=0$; that is they emerge from the two pinching pressures for mode 2 and mode 4 shapes respectively in static fluid (Flaherty et al. 1972). The left/right cell shapes inset into the figure correspond to $\hat{P}=55.0$ on branch $4 /$ solid part of branch 2 respectively. (b) Cell profiles at $\hat{P}=55$ at $\hat{\alpha}=0$ and $\hat{\alpha}=98.4$.

As $\hat{\alpha}$ is increased from zero, multiple solution branches emanate from the many possible states at $\hat{\alpha}=0$ summarised in the previous paragraph and in Table 1. Figure 5(a) shows the solution branch for the case $\hat{P}=3.2$. Parts of the branch extending to non-physical values $\hat{\alpha}<0$ are included to illustrate that the two apparently separate branches in $\hat{\alpha}>0$ are in fact connected. The whole solution branch has a cross-over point at $\hat{\mathscr{E}}=1.52$ at which there are two solutions for the cell shape relevant to our problem, that is two mode 2 shapes, one with major axis aligned with the oncoming stream and one with major axis perpendicular to the stream. Also shown in Figure 5(a) are typical cell shapes at three different points on the solution branch. At $\hat{\mathscr{E}}=1$ the cell is perfectly circular. According to the linear theory of section 3.2 the branch emanating from $\hat{\mathscr{E}}=1$ corresponds to mode 2 shapes with the major axis aligned with the horizontal. Cells of this type are found along the branch emanating from $\hat{\mathscr{E}}=1$ up to the branch corss-over point at $\hat{\mathscr{E}}=1.52$ (two typical cells are shown as shapes A and B). On the uppermost solution branch in Figure 5(a), the cell shape at $\hat{\alpha}=0$ is a partially buckled mode 2 shape at $\hat{\alpha}=0$ with major axis perpendicular to the oncoming stream. A typical cell further to the right along this branch is shown as shape C. Eventually, as $\hat{\alpha}$ is increased, cells along the uppermost branch reach a pinched state with a point of self-contact similar to that shown in Figure $2(b)$.

As was noted in section 3.2, a kind of resonance occurs at the critical pressure $\hat{P}=3$ in the limit $\hat{\alpha} \rightarrow 0$, where linear perturbation theory fails. The weakly nonlinear calculation presented in section 3.3 predicted the occurrence of a cusp in the $\hat{\mathscr{E}}-\hat{\alpha}$ curve at $\hat{\alpha}=0$, and this is confirmed by fully nonlinear calculations. As $\hat{P} \rightarrow 3^{+}$we find that the loop in Figure 5(a) shrinks and eventually disappears. The transition through $\hat{P}=3$ is shown in Figure 6, which demonstrates the change in character of the solution branch in $\hat{\mathscr{E}}-\hat{\alpha}$ space in the neighbourhood of the point $(\hat{\mathscr{E}}, \hat{\alpha})=(1,0)$ as $\hat{P}$ passes through 

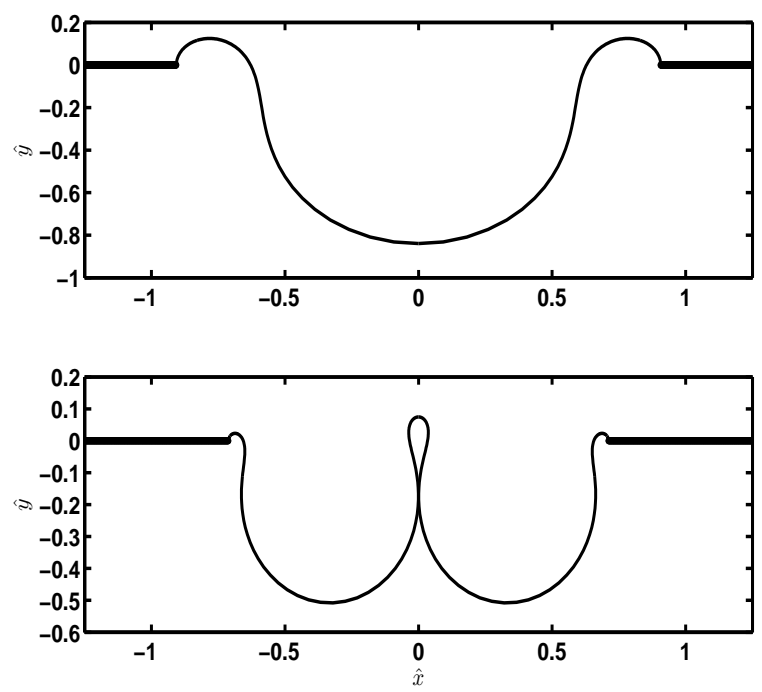

FIG. 8: Cell profiles straddling a wall aperture: $\hat{P}=3.2$ and $\hat{\alpha}=116.3$ (upper panel) and $\hat{P}=25$ and $\hat{\alpha}=7.74 \times 10^{3}$ (lower panel). The pinched cell profile in the lower panel appears eventually by continuing the solution branches seen in Figure $5(b)$ beyond point $B$. The heavy horizontal lines in each panel represent the solid wall.

3 from above. Parts of the solution branch in the non-physical parameter range $\hat{\alpha}<0$ are shown to aid mathematical understanding. Evidently the bifurcation diagram does indeed have a cusp at $\hat{\alpha}=0$ at the critical pressure $\hat{P}=3$. Moreover the quantitative agreement between the weakly nonlinear prediction for the bending energy (3.21), shown with crosses in the middle diagram, is excellent. Cells along the branch at $\hat{P}=3$ emanating from the cusp into $\hat{\alpha}>0$ are of mode 2 type with their major axis perpendicular to the oncoming stream, which is also in agreement with the weakly nonlinear theory prediction. Eventually, as $\hat{\alpha}$ increases, the cells reach a state with a point of self-contact.

In the range $5.247 \leqslant \hat{P} \leqslant 15$ the only relevant solution at $\hat{\alpha}=0$ is the circle according to Table 1 . Increasing $\hat{\alpha}$ leads to mode 2 shapes with major axis aligned with the oncoming stream, in agreement with the linear theory of section 3.2. Eventually $\hat{\alpha}$ reaches a critical value at which the cells pinch. In the range $15 \leqslant \hat{P} \leqslant 35$, the relevant solutions at $\hat{\alpha}=0$ are the circle and two mode 4 shapes which are equivalent to within a rotation through $\pi / 4$. Figure $5(b)$ shows the three solution branches which emerge from these three states at $\hat{\alpha}=0$. Solutions along each of these branches terminate at points corresponding to the pinched cell shapes $A, B$ and $C$. Comparing the solution space in this figure with that seen in Figure 5(b), we see that the loop containing $A, B$ has split into two disjoint parts.

Figure $7(a)$ shows the solution branches corresponding to pinched cell shapes. For $\hat{P}<22.3$ there is one pinched solution. This is found on the uppermost part of the branch in Figure 5(a), for example (recall that there are no pinched solutions on the loop through the points $A, B$ in this figure). When $\hat{P}>22.3$ two pinched solutions appear. In Figure $7(a)$, branch 1 corresponds to the cell shapes shown in Figure 3. Pinched cell shapes on branches 1, 2 (dashed part), 3 and 5 emerge from either a circle or from a mode 2 , mode 4 , or mode 6 buckled state as $\hat{\alpha}$ is increased from zero for constant $\hat{P}$. A cell profile which emerges as a continuous deformation from a mode 6 shape at $\hat{\alpha}=0$ is shown in Figure 


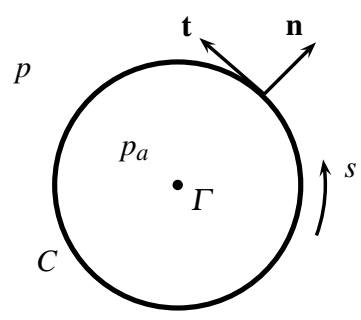

Fig. 8: Sketch of the elastic cell $C$ with a point vortex of strength $\Gamma$ at the centre. The fluid is outside.

$7(b)$. Branch 4 (shown with a solid line) and the solid part of branch 2 are particularly interesting as they correspond to pinched cell shapes which emerge from self-intersected cells as $\hat{\alpha}$ is increased from zero for fixed $\hat{P}$. This is to say that at certain values of $\hat{P}$, physically realisable cell shapes may be obtained in the presence of flow even when no physically admissible solutions exists at the same $\hat{P}$ in the absence of flow.

Finally we note that since an elastic cell may deform into a mode 2 shape with its major axis aligned with the flow, an alternative, and physically acceptable, interpretation of some of the self-intersecting cell shapes which are found beyond the point of contact is available. Referring to the sketch in Figure 1(a), we disregard that part of the flow-field in $y<0$ and view the sections $A B$ and $C D$ of the $x$-axis as a solid wall. The upper part of the cell may be then be viewed as an elastic cap over an aperture into a region of constant pressure. Two examples are shown in Figure 8. The configuration shown in the lower panel of this figure corresponds to a point on the continuation of the branch through point $B$ in Figure $5(b)$ beyond the point of self-contact. Note that a similar intrepretation is not possible for a bubble since this always deforms into a mode 2 shape with major axis perpendicular to the stream.

\section{An elastic cell in a circulatory flow}

In this section we study the shape of an elastic cell placed into a circulatory flow, as is illustrated in Figure 8. The circulatory flow in the fluid, which lies in the infinite region outside the cell, is induced by a point vortex inside the cell. In the unperturbed configuration the cell boundary $C$ is circular, of radius $a$, and the point vortex is located at the cell centre $z=0$. Our goal is to identify non-circular equilibrium states. The complex potential associated with the point vortex when viewed in isolation is given by

$$
w=-\mathrm{i} \frac{\Gamma}{2 \pi} \log (z / a),
$$

where $\Gamma$ is the strength of the vortex and $z=x+\mathrm{i} y$. For $\Gamma>0$ the fluid swirls around the cell in the anticlockwise direction. Selecting the length scale $L=a$ and the velocity scale $U=\Gamma / a$, the dimensionless parameter in (2.5) becomes

$$
\alpha=\frac{\rho a \Gamma^{2}}{E_{B}} .
$$

In order to analyse the cell deformation in response to the vortex-induced flow, we write (e.g. 
Vanden-Broeck 2010)

$$
\frac{\mathrm{d} w}{\mathrm{~d} z}=u-\mathrm{i} v=\mathrm{e}^{\tau-\mathrm{i} \theta}
$$

where $u, v$ are the velocity components at $C$ in the $x, y$ directions respectively. Here $\tau=\log \left(u^{2}+v^{2}\right)^{1 / 2}$, and $\theta$ is the angle between the unit tangent vector $\mathbf{t}$ (shown in Figure 8 ) and the horizontal.

To represent the flow, we aim to find an analytic function $\tau-\mathrm{i} \theta$ such that the dynamic condition (2.4) and the no-penetration condition hold on the boundary $C$. For computational purposes, we note that (Blyth et al. 2012)

$$
\kappa=-\mathrm{e}^{\tau} \frac{\partial \theta}{\partial \phi}, \quad \kappa^{\prime \prime}=-\mathrm{e}^{3 \tau}\left[2\left(\frac{\partial \tau}{\partial \phi}\right)^{2} \frac{\partial \theta}{\partial \phi}+3 \frac{\partial \tau}{\partial \phi} \frac{\partial^{2} \theta}{\partial \phi^{2}}+\frac{\partial^{2} \tau}{\partial \phi^{2}} \frac{\partial \theta}{\partial \phi}+\frac{\partial^{3} \theta}{\partial \phi^{3}}\right] .
$$

For the undeformed, circular cell boundary we have $\kappa=-1$.

Insisting that the boundary $C$ corresponds to a streamline on which $\psi=0$, we transform the flow domain in the complex $z$-plane into the interior of the unit circle in the $\zeta$-plane via the mapping

$$
\zeta=\mathrm{e}^{-2 \mathrm{i} \pi w}
$$

note that $|\zeta|=1$ on $\psi=0$. We seek a solution by writing (Vanden-Broeck \& Miloh 1995)

$$
\tau-\mathrm{i} \theta=A+g(\zeta), \quad A=-\log 2 \pi-\mathrm{i} \pi / 2+\log \zeta,
$$

where $A$ represents the singular contribution made by the point vortex (4.1) and $g(\zeta)$ is a function which is analytic inside the unit circle. For $g(\zeta)$ we seek the series representation

$$
g(\zeta)=\sum_{n=1}^{\infty} a_{n} \zeta^{n}
$$

where the $a_{n}$ are coefficients to be found. Assuming that the cell boundary has a line of reflectional symmetry, a straightforward argument shows that the $a_{n}$ are real. When $C$ has $k$-fold rotational symmetry we find that

$$
a_{n}=0 \quad \text { if } \quad n \neq 0(\bmod k) .
$$

For an undeformed, circular cell boundary $C$ all of the coefficients $a_{n}$ are zero. The task is now to compute the coefficients $a_{n}$ to satisfy the dynamic boundary condition (2.4). In general this must be done numerically, and a suitable method will be described in due course. First, in the next section, we consider small amplitude cell deformation.

\subsection{Linear theory}

The velocity potential for the flow with circular streamlines around the undeformed circular boundary $C$ is given by $\phi=\theta / 2 \pi$. Denoting the dimensionless polar distance to the centre of the undeformed boundary by $r$, we perturb $C$ to the new configuration described by

$$
r=1+\varepsilon \cos k \theta
$$


where $k$ is an integer constant and $\varepsilon \ll 1$ is a small parameter. We note that under this perturbation the perimeter of the boundary $C$ is unchanged up to $O\left(\varepsilon^{2}\right)$. Ignoring contributions of size $O\left(\varepsilon^{2}\right)$, the choice $k=1$ simply corresponds to a small translation of $C$ through a distance $\varepsilon$ which preserves the unperturbed circular shape, and for this reason we restrict attention to $k \geqslant 2$. Consistent with (4.9) the basic flow is perturbed so that the velocity potential becomes

$$
\phi=\frac{\theta}{2 \pi}+\varepsilon \Phi(r) \sin k \theta
$$

where the function $\Phi(r)$ is to be determined. Computing the perturbed velocity field and substituting into the dynamic boundary condition (2.4), we obtain at the leading order,

$$
\frac{\alpha}{8 \pi^{2}}-\left(\sigma-\frac{1}{2}\right)=B,
$$

and at $O(\varepsilon)$ we find

$$
\frac{\alpha}{2 \pi}\left(k \Phi(1)-\frac{1}{2 \pi}\right)-\left(1-k^{2}\right)\left(\frac{3}{2}-\sigma-k^{2}\right)=0 .
$$

At first order, the kinematic condition on the elastic boundary requires

$$
\Phi^{\prime}(1)=-\frac{k}{2 \pi}
$$

The solution for $\Phi$ which remains regular at infinity and which satisfies (4.13) is $\Phi=r^{-k} /(2 \pi)$. Substituting this into (4.12) and using (4.11) we arrive at the dispersion relation

$$
\alpha=8 \pi^{2}\left(\frac{k+1}{k-1}\right)\left(B-\left(k^{2}-1\right)\right) .
$$

Wegmann \& Crowdy (2000) derived a similar dispersion relation for the case of bubble with surface tension in a circulatory flow. It is interesting to note that the dispersion relation (4.14) requires $B>k^{2}-1$ for positive $\alpha$, which parallels the condition on the transmural pressure for a buckled tube in a static liquid (see the comments at the end of section 2).

\subsection{Numerical method}

In our numerical method we truncate the series in (4.7) at a finite level and determine the series coefficients via a collocation method. In view of the symmetry conditions (4.8), we may re-express the infinite series (4.7) at a point on the boundary in the form

$$
g(\zeta)=\sum_{l=1}^{\infty} b_{l} \zeta^{k l}=\sum_{l=1}^{\infty} b_{l} \mathrm{e}^{-2 \mathrm{i} \pi k l \phi}
$$

where $b_{l}=a_{k l}$, and we have used the fact that $\zeta=\exp (-2 \mathrm{i} \pi \phi)$ on $C$ for $0 \leqslant \phi \leqslant 1$. Due to the $k$-fold rotational symmetry and the reflectional symmetry we need only collocate the portion $0 \leqslant \phi \leqslant 1 /(2 k)$. We place $N$ collocation points on the elastic boundary at locations denoted by the values of the velocity potential,

$$
\phi_{j}=\frac{(j-1)}{N} h, \quad j=1, \ldots, N
$$


where $h=1 /(2 k)$. By applying (2.4) at each of the collocation points, we complete a set of $N$ nonlinear algebraic equations for the $N$ unknowns including $b_{l}, l=1, \ldots, N-1$, and the Bernoulli constant $B$. The equations are solved iteratively using Newton's method and a suitable initial guess. In particular when tracing solution branches it is convenient to apply a continuation method and use as initial guess the converged solution from a previous calculation.

In computational practice we followed two different approaches: first to choose values of $\alpha$ and $\sigma$ and to solve for the set $b_{l}, l=1, \ldots, N-1$ and $B$, and second to choose values of $\sigma$ and $b_{1}$ and to solve for $b_{l}, l=2, \ldots, N-1$, and $B$ and $\alpha$. The second approach is convenient to ensure that a deformed, noncircular solution is obtained and is consistent with the discussion in section 4.1 concerning linear theory, where we effectively fix the amplitude of the wave and then fix one of the three parameters $\alpha, B$ and $\sigma$. Once a solution has been found, its physical profile in the $x-y$ plane is constructed by integrating the identity

$$
\frac{\partial x}{\partial \phi}+\mathrm{i} \frac{\partial y}{\partial \phi}=\frac{1}{u-\mathrm{i} v}=\mathrm{e}^{-\tau+\mathrm{i} \theta}
$$

over the range $0 \leqslant \phi \leqslant 1 /(2 k)$ and using the symmetry properties to assemble the remainder of the cell profile.

As a check on the numerics we compare with the predictions of linear theory discussed in section 4.1 for the sample case $\sigma=0$. For $k=2$ the linear theory yields $\alpha=30 \pi^{2} \approx 296$ and $B=17 / 4=4.25$, and the numerical calculations yield $\alpha=296$ and $B=4.25$ and so both sets of values agree to the given precision. Higher values of $k$ were also checked with similar success.

\subsection{Results}

Having computed a deformed cell shape we can calculate its dimensionless perimeter, $\lambda$, using the formula

$$
\lambda=\int_{0}^{1} \mathrm{e}^{-\tau} \mathrm{d} \phi
$$

In presenting our results we prefer to discuss how a circulatory flows of different strengths affect the same elastic cell of fixed perimeter. To this end, we note that (2.4) is invariant under the set of transformations

$$
(u, \kappa, \alpha)=\left(\frac{2 \pi}{\lambda}\right)(\hat{u}, \hat{\kappa}, \hat{\alpha}), \quad s=\left(\frac{\lambda}{2 \pi}\right) \hat{s}, \quad \sigma=\left(\frac{2 \pi}{\lambda}\right)^{2} \hat{\sigma}, \quad B=\left(\frac{2 \pi}{\lambda}\right)^{3} \hat{B} .
$$

Applying the transformations (4.19) effectively corresponds to redefining the original choice of scales used in the non-dimensionalisation from $a$ to $\lambda a$ for the length and from $\Gamma / a$ to $\Gamma /(\lambda a)$ for the velocity. In so doing we may take our computed solutions and rescale so that $(\hat{x}, \hat{y})=(2 \pi / \lambda)(x, y)$ to obtain the solution in the $\hat{x}-\hat{y}$ plane for a cell of perimeter $2 \pi$. This aids comparison with the work of Flaherty et al. (1972) who discuss cells of dimensionless perimeter $2 \pi$. To describe our solutions it is convenient to refer to the dimensionless measure of the bending energy in the cell boundary,

$$
\hat{\mathscr{E}}=\frac{1}{2 \pi} \int_{0}^{2 \pi} \hat{\kappa}^{2} \mathrm{~d} \hat{s}
$$

For an undeformed circular cell $\hat{\mathscr{E}}=1$. 
(a)

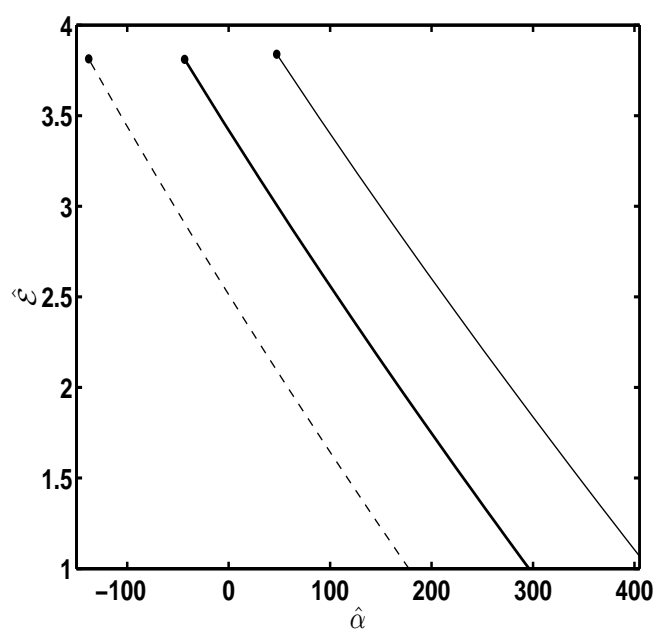

(b)

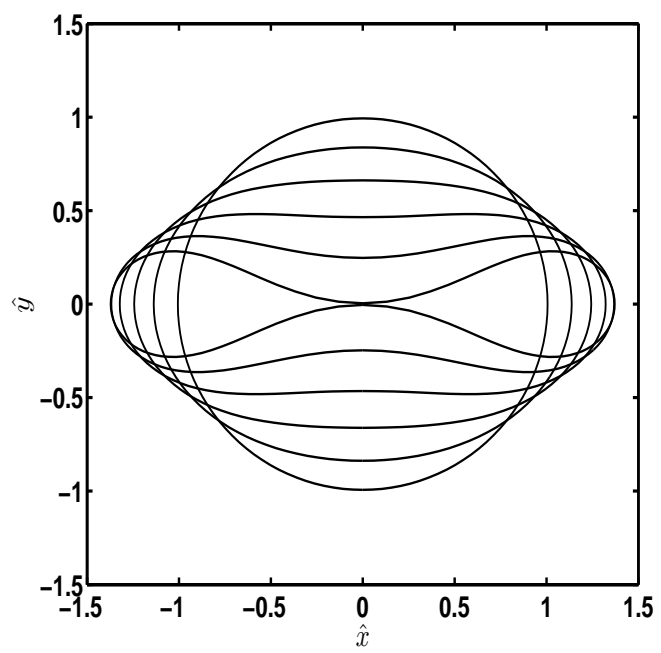

FIG. 10: (a) Mode $k=2$ solution branches for $\hat{\sigma}=0$ (thick solid line), $\hat{\sigma}=1$ (thin solid line) and $\hat{\sigma}=-1$ (dashed line). The cells are circles at $\hat{\mathscr{E}}=1$ on each branch and reach profiles with points of self-contact at the dots at the end of the branches.

(a)

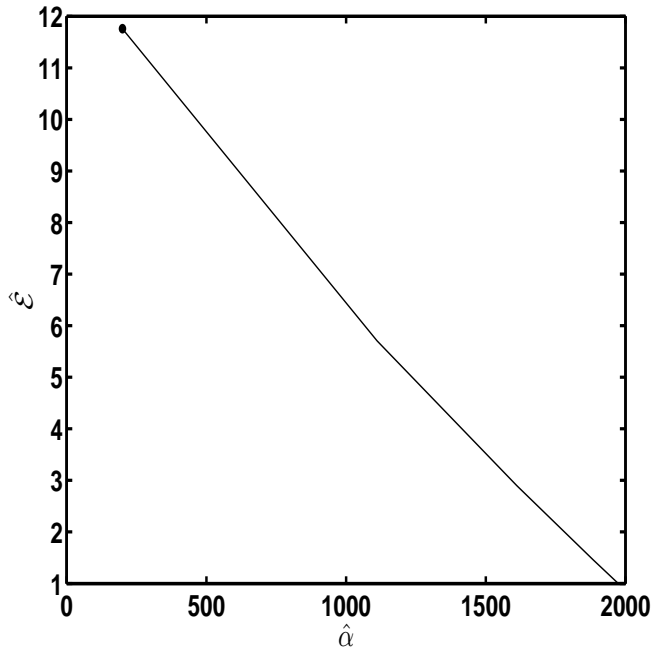

(b)

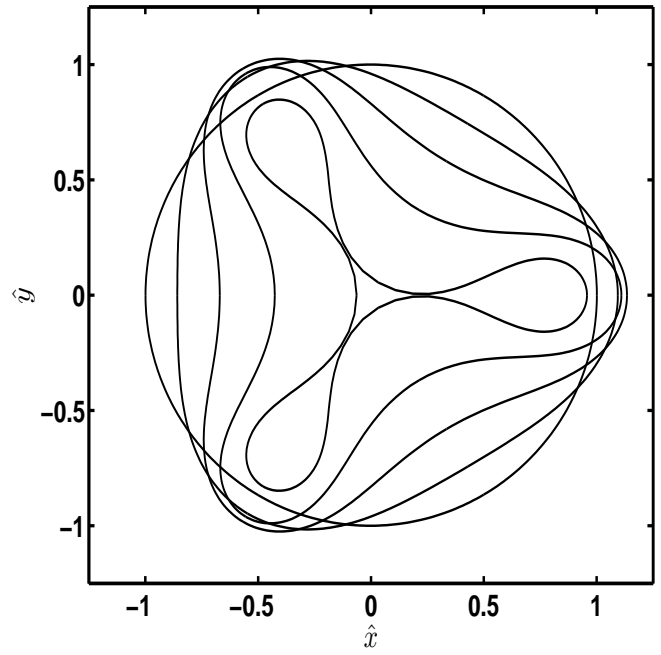

FIG. 11: (a) Mode $k=3$ solution branches for $\hat{\sigma}=5$. The cell is a circle at $\hat{\mathscr{E}}=1$ and reaches a profile with a point of self-contact at the dot at the end of the branch. 
In Figure 10 we show mode $k=2$ solution branches for three different values of $\hat{\sigma}$. Traversing each of the branches from left to right the cells deform from circles (with $\hat{\mathscr{E}}=1$ ) into shapes with a point of self-contact. The solution branches may be continued further to obtain physically implausible self-intersecting cell-profiles. Evidently solutions whose cell profiles do not self-intersect, but for which $\hat{\alpha}$ is negative, are possible although it is not clear how to interpret these in a physically meaningful way - the formulation of our problem sensibly demands that $\hat{\alpha}$ be positive. Mode $k=3$ solutions are shown in Figure 11 for the case $\hat{\sigma}=5$. This value was chosen so that the profile with a point of self-contact is reached in the physically realisable $\hat{\alpha}$ positive regime.

When $\hat{\alpha}=0$ the dynamic condition at the cell boundary, namely (2.4), reduces to the equation describing the shape of a cell held in a constant pressure environment studied by Flaherty et al. (1972). In this case our $B$ corresponds to their pressure $p$ and our constant $\hat{\sigma}$ corresponds to their constant $c$ (notice that they define curvature to be positive for the circular state whereas we take it to be negative). We have confirmed that when our solution branches meet the line $\hat{\alpha}=0$ we faithfully recompute the pertinent solutions found by Flaherty et al. (1972). In fact, we recomputed their solutions independently and checked that our value of $B$ for a chosen $\hat{\sigma}$ corresponded exactly to their values of $p$ and $c$ respectively (note that they do not explicitly report the value of $c$ ).

\section{Summary}

We have studied the deformation of an inextensible thin-walled elastic cell placed in either a uniform stream or a circulatory flow. Using complex variable methods we have computed the cell configurations which arise in these two scenarios for different flow strengths. Our work extends the calculations of Flaherty et al. (1972) for buckled cells in a quiescent environment at constant pressure to the case where deformation occurs in response to a spatially-dependent dynamic pressure at the cell boundary. For a cell placed into a uniform stream, the background flow forces upon a circular cell a mode- 2 type deformation. A key result of our work is the observation that while a bubble with constant surface tension must deform linearly in a weak uniform stream into an ellipse with its minor axis aligned with the flow, for an elastic cell the linearly deformed shape is again an ellipse but either its minor or its major axis may be aligned with the flow. While mode- 2 type deformation dominates for small deformations from a circular state, higher order modes can come into play, and indeed dominate the cell shape, under strong deformation. In all of our calculations for a cell in a uniform stream, we have assumed that cells have a horizontal line of symmetry. The issue of whether cell shapes which are not up-down symmetric are possible has not been addressed here and is left as a problem for future work. For a cell placed into a circulatory flow, no single mode shape dominates and cells with $m$-fold rotational symmetry are possible.

The stability of the equilibrium shapes computed here has not been addressed and this important issue is left as a problem for future work. Another possible avenue for extension is to compute waves on a fluid annulus bounded by elastic plates, the capillary version of which has been studied by Crowdy (1999b) and Blyth \& Vanden-Broeck (2005). Perhaps the most natural extension of the present work is to consider the combination of our two flow-fields, namely the flow past an elastic cell endowed with a finite circulation. This would provide a simple model of the flow past a flexible aerofoil. Work on this problem is currently underway.

Acknowledgements We would like to thank an anonymous referee for very helpful suggestions and constructive comments on the manuscript. EIP thanks EPSRC for support under grant EP/J019305/1. 
Note: This is a pre-copy-editing, author-produced PDF of an article accepted for publication in the IMA Journal of Applied Mathematics following peer review. The definitive publisher-authenticated version M.G. Blyth, E.I. Parau, Deformation of an elastic cell in a uniform stream and in a circulatory flow, IMA J. Applied Maths.78(2013), 665-684. DOI: 10.1093/imamat/hxt014 is available online at: http://imamat.oxfordjournals.org/content/78/4/665.

\section{References}

AlBEN, S. (2011) Interactions between vortices and flexible walls, Int. J. Nonlin. Mech., 46, 586-591.

Antman, S. S. 2005 Nonlinear problems in elasticity, Springer, New York.

Batchelor, G. K. 1967 An introduction to fluid dynamics. Cambridge University Press, Cambridge.

Blyth, M. G., PĂRĂU, E. I. \& VANDEN-BRoeck, J-M. 2011 Hydroelastic waves on fluid sheets, J. Fluid Mech., 689, 541-551.

BLyth, M. G. \& VANDEN-BRoecK, J-M. 2005 New solutions for capillary waves on curved sheets of fluid, IMA J. Appl. Math., 70, 588-601.

CRAPPER, G. D. 1957 An exact solution for progressive capillary waves of arbitrary amplitude, $J$. Fluid Mech. 2, 532-540.

Crowdy, D. 1999a Circulation-induced shape deformations of drops and bubbles: Exact twodimensional models, Phys. Fluids, 11, 2836-2845.

CROwdy, D. 1999b Exact solutions for capillary waves on a fluid annulus, J. Nonlinear Sci., 9, 615640.

Crowdy, D. 2001 Steady nonlinear capillary waves on curved sheets, Eur. J. Appl. Math., 12, 689708.

DRAZIN, P. G. 1992 Nonlinear systems. Cambridge University Press, Cambridge.

Flaherty, J. E., Keller, J. B. \& Rubinow, S. I. 1972 Post buckling behaviour of elastic tubes and rings with opposite sides in contact SIAM J. Appl. Math. 23, 446-455.

FUnG, Y. C. 1965 Foundations of solid mechanics, Prentice, New York.

FunG, Y. C. 1997 Biomechanics: Circulation, Springer, New York.

Korobkin, A., PĂrăU, E. I. \& VAnden-Broeck, J-M. 2011 The mathematical challenges and modelling of hydroelasticity, Phil. Trans. Roy. Soc. 369, 2803-2810.

Lauga, E. \& Powers, T. R. 2009 The hydrodynamics of swimming microorganisms, Rep. Prog. Phys., 72, 096601.

MCLEOD, E. B. 1955 The explicit solution of a free boundary problem involving surface tension, $J$. Rat. Mech. Anal., 4, 557-567.

Michelin, S. \& Llewellyn Smith, S. G. 2010 Falling cards and flapping flags: understanding fluid-solid interactions using an unsteady point vortex model, Theor. Comp. Fluid Dyn., 24, 195-200. 
Ozugurlu, E. \& VAnden-Broeck, J-M. 2000 The distortion of a bubble in a corner flow, Euro. J. Appl. Math. 11, 171-179.

Petrova, S. I. 1971 Equilibrium shape of a cavity bounded by an elastic film in a homogeneous flow of a liquid, Fluid Dyn., 6, 104-110.

PoZRIKIDIS, C. 2002 Buckling and collapse of open and closed cylindrical shells, J. Engng. Math., 42, 157-180.

Ten, I., Malenica, S̆. \& KorobKin, A. 2011 Semi-analytical models of hydroelastic sloshing impact in tanks of liquefied natural gas vessels, Phil. Trans. Roy. Soc. 369, 2920-2941.

Vanden-Broeck, J-M. \& Keller, J. B. 1980a A new family of capillary waves, J. Fluid Mech. 98, 161-169.

VAnden-Broeck, J-M. \& Keller, J. B. 1980b Deformation of a bubble or drop in a uniform flow. J. Fluid Mech. 101, 673-686.

VAnden-Broeck, J.-M. \& Miloh, T. 1995 Computations of steep gravity waves by a refinement of Davies-Tulins approximation, SIAM J. Appl. Maths, 55, 892903

VAnden-Broeck, J-M. 2010 Gravity-capillary free surface flows, Cambridge University Press, Cambridge.

WEGMANN \& CROWDY, D. 2000 Shapes of two-dimensional bubbles deformed by circulation, Nonlinearity, 13, 2131-2141. 